\title{
ist \\ Un nuevo esquema conceptual para la interpretación de las mezclas impropias en mecánica cuántica
}

\author{
Federico Holik \\ Christian DE RONDE \\ Wim Ghristiaens
}

\begin{abstract}
$\stackrel{\sim}{\dddot{2}}$
RESUMEN

En este artículo, analizamos el significado de las matrices densidad en el formalismo de la mecánica cuántica. Discutimos el problema de los "sistemas cuánticos compuestos" en la lógica cuántica así como también la interpretación de las mezclas impropias. Tomando en cuenta el desarrollo de la lógica cuántica convexa, presentamos un análisis de la estructura formal de la teoría que, argumentaremos, debe ser considerado a la hora de desarrollar un nuevo esquema conceptual para la interpretación de las mezclas cuánticas.
\end{abstract}

Palabras-GLAVE • Lógica cuántica. Mezclas impropias. Conjuntos convexos.

\section{INTRODUCGIÓN}

Desde sus inicios, la mecánica cuántica ha sido fuente de grandes debates filosóficos. Uno de los más importantes en el contexto de la filosofía de la ciencia es el debate realismo/anti-realismo, para el cual la teoría cuántica ha sido utilizada como campo de batalla propicio para argumentar a favor de una y otra posición. La postura realista podría enunciarse del siguiente modo: hay una realidad externa que existe en si misma, que es independiente de la conciencia de los sujetos y puede ser conocida. Usualmente las posiciones realistas presuponen un esquema metafísico clásico, constituido por objetos cuyas propiedades obedecen los principios de la lógica clásica. Por otro lado, la postura anti-realista afirma que las teorías físicas no describen la realidad, sino que son meras "estructuras útiles" para la organización de la experiencia. Según se entiende en la literatura de la filosofía de la ciencia, en la mecánica cuántica, ese debate tuvo su expresión más acabada en las discusiones que tuvieron lugar entre Niels Bohry Albert Einstein en las primeras décadas del siglo xx. Como señala, por ejemplo, Arthur Fine, 
"la guerra entre Einstein, el realista, y Bohr, el no-realista, respecto de la interpretación de la teoría cuántica no era, creo yo, simplemente un espectáculo dentro de la física, ni un ejercicio intelectual ocioso. Fue un esfuerzo importante realizado por Bohr en nombre de la empresa de la física como una ciencia progresiva." (Fine, 1986, p. 88). ${ }^{1}$ Si bien no creemos que Einstein pueda ser considerado "realista", por lo menos no en el sentido en que se utiliza el término en la filosofía de la ciencia (cf. Howard, 1993; 1994), resulta interesante de igual modo tomar en cuenta el debate a partir de lo que estos autores discuten respecto de la referencia al mundo por parte de las teorías físicas. Lo cierto es que la mecánica cuántica ha desafiado desde sus orígenes a las posiciones realistas, lo que se debe fundamentalmente a que su estructura formal parece ir en contra de los presupuestos lógicos a partir de los cuales es posible concebir los "objetos clásicos". Es a partir de ese límite que el problema de la interpretación de la mecánica cuántica cuenta con una riquísima historia que continúa hasta nuestros días. Sin embargo, como señala Arthur Fine, debemos también tomar en cuenta que ha sido la mecánica cuántica la teoría que, siguiendo los lineamientos metodológicos de Niels Bohr, ha tenido una influencia insoslayable a la hora de desarrollar posiciones eminentemente anti-metafísicas.

En el cuerpo del artículo [de 1925], Heisenberg no sólo rechaza cualquier referencia a los no-observables, sino que también sostiene la idea de que uno no debe tratar de formarse una imagen de la realidad subyacente a su mecánica. Sin duda, Schrödinger, el segundo padre de la teoría cuántica, parece originalmente haber sostenido una vaga imagen, de una realidad ondulatoria, subyacente a su ecuación de ondas. Pero, al ver rápidamente las dificultades que tal visión presentaba, con la misma rapidez, aunque de mala gana, abandonó el intento de interpolar cualquier referencia a la realidad. Esos movimientos instrumentistas, lejos de una construcción realista de la naciente teoría cuántica, tuvieron un empuje importante a partir de la llamada "filosofía de la complementariedad" propuesta por Bohr; esa posición anti-realista se consolidó en la famosa conferencia de Solvay, en octubre de 1927, y está firmemente en su lugar hoy. Tal anti-realismo cuántico es parte de lo que todo físico graduado aprende y práctica. Es el telón de fondo conceptual para todos los éxitos brillantes en la física atómica, nuclear y de partículas en los últimos cincuenta años. Los físicos han aprendido a pensar acerca de su teoría en forma no realista, y haciendo precisamente esto han traído el éxito predictivo más maravilloso en la historia de la ciencia (Fine, 1986, p. 88).

1 Aquí como en el resto del artículo las traducciones nos corresponden. 
UN NUEVO ESQUEMA GONGEPTUAL PARA LA INTERPRETAGióN...

Dejando de lado las posiciones anti-metafísicas que sostienen la irrelevancia de una interpretación de la mecánica cuántica, ${ }^{2}$ podemos considerar el amplio espectro en que se ubican las diversas interpretaciones a partir de la estrategia interpretativa en que se desarrollan. En este sentido uno de los autores del presente trabajo ha discutido la posibilidad de distinguir esencialmente entre dos estrategias (cf. de Ronde, 2010). La primera: comenzar por un esquema metafísico y avanzar en el desarrollo de un nuevo formalismo de la teoría - como es el caso, por ejemplo, de la interpretación de nocolapso de Bohm (cf. 1952a, 1952b) o la interpretación de colapso de Ghirardi, Rimini y Weber (cf. 1986) -; la segunda estrategia, a saber, comenzar por el formalismo ortodoxo y buscar un esquema metafísico coherente que permita entenderlo, ha sido sugerida e investigada por las diversas interpretaciones de la lógica cuántica entre las que se encuentran, por ejemplo, la interpretación modal (cf. Dickson \& Dieks, 2002; Kochen, 1985; van Fraassen, 1991) y el enfoque de Ginebra (cf. Jauch, 1968; Piron, 1976).

En este trabajo, nos ubicamos dentro del marco impuesto por esta segunda estrategia, en la cual aparece como necesario el análisis del formalismo de la mecánica cuántica por un lado, y el desarrollo de nuevos esquemas conceptuales por otro. Siguiendo esta línea de pensamiento nos ocuparemos de reconsiderar el lugar que ocupan las matrices densidad dentro de la teoría. Más específicamente, analizaremos el significado e interpretación que puede darse a las matrices densidad en el formalismo ortodoxo de la mecánica cuántica. Discutiremos además el problema de los 'sistemas cuánticos compuestos' en la lógica cuántica así como también la interpretación de las denominadas mezclas impropias (cf. D’Espagnat, 1976, cap. 6). Tomando en cuenta el desarrollo de la lógica cuántica convexa (cf. Holik, Massri \& Ciancaglini, 2012), presentaremos un análisis de la estructura formal de la teoría que creemos puede resultar apropiado para desarrollar, a partir de nuevos análisis formales, un nuevo esquema conceptual para la interpretación de las mezclas cuánticas. En la sección 1, discutiremos el formalismo de la mecánica cuántica en su versión ortodoxa para estados puros. En la sección 2, consideraremos, tomando en cuenta los sistemas compuestos, las mezclas cuánticas que surgen a partir de las trazas del sistema total como estados reducidos. Analizaremos también los problemas interpretativos y formales que surgen a partir de tal desarrollo. La sección 3 provee una solución formal de la relación entre sistemas y subsistemas en la mecánica cuántica a partir de la lógica cuántica convexa.

2 Por ejemplo, Christopher Fuchs y Asher Peres han señalado que “(...) la mecánica cuántica no describe la realidad física. Lo que hace es proveer un algoritmo para calcular las probabilidades para los eventos macroscópicos (clicks en los detectores) que son consecuencia de las intervenciones experimentales. Esa definición estricta del alcance de la teoría cuántica será la única interpretación necesaria que deberán utilizar tanto físicos experimentalistas como teóricos." (2000, p. 70). 
Finalmente, en la sección 4, analizamos algunas consideraciones que, argumentaremos, deben ser tomadas en cuenta a la hora de desarrollar un nuevo esquema conceptual que permita interpretar coherentemente las matrices densidad dentro del esquema formal ortodoxo de la teoría.

\section{RefleXiones En toRno AL Formalismo dE LA MECÁNICA CUÁNTICA}

En otro lugar, hemos considerado a las teorías físicas como constituidas a partir de una tríada: estructura matemática, estructura conceptual y experiencia física pensable (cf. de Ronde, 2009; 2011). Siguiendo a Heisenberg, sostenemos que cada teoría física debe ser en última instancia una teoría cerrada ${ }^{3}$ Cada una de esas teorías cerradas se encuentra determinada por conceptos y expresiones matemáticas que permiten considerar y pensar un campo de fenómenos con el objeto de llegar a la experiencia particular y singular, hic et nunc. En el caso de la física clásica, la estructura matemática se encuentra dada por el espacio de fases y su estructura simpléctica mientras que la estructura conceptual es la de las entidades u objetos físicos, determinados en términos lógicos a partir de los principios de existencia, no-contradicción e identidad. La experiencia pensada es, entonces, aquélla que nos permite concebir que un cuerpo evoluciona a partir de ciertas condiciones iniciales en el espacio-tiempo. Existe, por así decirlo, un esquema coherente que permite tanto pensar como predecir un conjunto de fenómenos físicos bien determinados. Sin embargo, en el caso de la mecánica cuántica, nos enfrentamos con una paradoja respecto del sentido físico de la teoría, puesto que si bien tenemos un esquema matemático - dado por la estructura formal en el espacio de Hilbert - y parecerían existir también un conjunto de experiencias que podemos predecir unívocamente a partir de esa estructura, no somos capaces en forma coherente de dar cuenta de qué es aquello respecto de lo cuál nos habla la teoría. Como es bien sabido, la estructura formal ortodoxa encuentra un sin número de inconvenientes a la hora de pensar la existencia de objetos con propiedades bien determinadas (cf. Bitbol, 1997; Mittelstaedt, 1998; de Ronde \& Bontems, 2011).

Los estados puros ${ }^{4}$ pueden ser representados en la mecánica cuántica por vectores en el espacio de Hilbert. Una de las preguntas centrales que debe responder cualquier interpretación que busque brindar una referencia conceptual a la teoría es: ¿qué

3 Según Heisenberg, las teorías cerradas se encuentran determinadas por un conjunto interrelacionado de conceptos, definiciones y leyes que agrupan y describen un gran conjunto de fenómenos. Para una discusión pertinente, cf. Bokulich, 2004; de Ronde \& Fleisner, 2012.

4 Los estados puros se encuentran dados por una matriz densidad $\rho$ y cumplen que: $\rho=\rho^{2}$, es un operador hermítico, positivo y de traza uno. 
significa que el estado puro de un sistema se encuentra representado por un vector $|\varphi\rangle$ en el espacio de Hilbert? (cf. de Ronde 2011, sección 7.1) El espacio de Hilbert es un espacio vectorial cerrado por las combinaciones lineales, lo cual significa que dados dos vectores normalizados, $|\mu\rangle$ y $|v\rangle$, que representan estados físicos, siempre podemos tomar coeficientes á y â, para formar una combinación lineal y obtener un nuevo estado $|\lambda\rangle$ a partir de ellos:

$$
|\lambda\rangle=\alpha|\mu\rangle+\beta|v\rangle
$$

Según Dirac, es este principio, no sólo el más importante de la teoría, sino también aquel que determina una distancia insalvable respecto de las teorías clásicas.

La naturaleza de las relaciones que el principio de superposición requiere que existan entre los estados de cualquier sistema es de un tipo que no puede ser explicado en términos de conceptos físicos familiares. En el sentido clásico, uno no puede imaginar un sistema parcialmente en dos estados y ver esto como equivalente a que el sistema se encuentre en otro estado. Hay involucrada una idea completamente nueva, a la que hay que acostumbrarse y en función del cual se debe proceder a construir una teoría matemática exacta, sin contar con una imagen clásica detallada (Dirac, 1974, p. 12).

La estructura del espacio de Hilbert implica a su vez que, dado cualquier estado representado por un vector $|\psi\rangle$, es posible expresar dicho estado como una combinación lineal de una infinidad de vectores que pertenecen a los sub-espacios de los que $|\psi\rangle$ es un elemento:

$$
|\psi\rangle=\Sigma \alpha_{\mathrm{i}}\left|\psi^{\prime}\right\rangle=\Sigma \alpha^{\prime \prime}{ }_{\mathrm{i}}\left|\psi^{\prime \prime}\right\rangle=\Sigma \alpha^{\prime \prime}{ }_{\mathrm{i}}\left|\psi^{\prime \prime}\right\rangle=\ldots
$$

donde $\left\{\left|\psi_{i}\right\rangle\right\},\left\{\left|\psi^{\prime}\right\rangle\right\},\left\{\left|\psi^{\prime}\right\rangle\right\} \ldots$, representan un conjunto infinito de bases orto-normales y los $\alpha_{i}{ }^{\prime}, \alpha_{i}{ }^{\prime}, \alpha_{i}{ }^{\prime \prime}$, , .. son las coordenadas de $|\psi\rangle$ en las distintas bases. Evidentemente, desde el punto de vista formal, no puede determinarse ninguna base como "preferencial", y debido a la incompatibilidad entre las bases y la no-conmutatividad de los observables no es posible tampoco considerar a las bases como representando propiedades preexistentes de un uno y mismo objeto. Un contexto se encuentra determinado por un arreglo experimental que en general se relaciona con un "conjunto completo de observables que conmutan” (CCOC). 5 La contextualidad cuántica muestra que, 
si bien es posible pensar éste o aquél experimento, así como el conjunto de propiedades que se derivan de ellos, existe una imposibilidad de orden formal para pensar simultáneamente la existencia de las propiedades del sistema independientemente de la representación del experimento. Este problema fue discutido primeramente por Bohr y Einstein en referencia al experimento de la doble rendija, experimento que muestra que no es posible considerar como existentes en forma simultánea a las propiedades derivadas de las efectuaciones expuestas en términos de la "representación de onda" y de la "representación de partícula". En términos de la lógica cuántica (cf. Birkhoff \& von Neumann, 1936), la contextualidad se manifiesta en el hecho de que mientras para sistemas clásicos la estructura lógica del álgebra de propiedades de un sistema físico resulta booleana, el álgebra de propiedades de un sistema cuántico determina una estructura ortomodular donde no se cumple la distributividad. En el mismo sentido, el teorema de Kochen-Specker (cf. 1967) muestra explícitamente que en el formalismo cuántico ortodoxo no es posible asignar una familia de valuaciones consistentes a las proposiciones acerca de las propiedades de un sistema cuántico independientemente del contexto.

La noción de contextualidad en la teoría cuántica resulta un concepto completamente extraño a las teorías de la física clásica, puesto que la idea de que un sistema cuántico pueda manifestar propiedades mutuamente incompatibles, dependiendo de la elección del COCC, se encuentra en completa contradicción con la presuposición clásica de discutir respecto de un objeto físico prexistente a la medición de sus propiedades actuales. Todos estos inconvenientes derivados del formalismo de la teoría deben ser considerados a la hora de discutir las posibilidades de una interpretación. En la próxima sección, veremos como la incompatibilidad de la mecánica cuántica con los conceptos clásicos se hace inclusive más evidente a partir del estudio de los llamados "sistemas compuestos" en donde el uso de mezclas impropias resulta inevitable.

\section{El CONJUNTO CONVEXo de ESTAdos GUÁNTicos}

Como hemos señalado anteriormente, entendemos que el problema fundamental de la mecánica cuántica es que no parece haber una red conceptual adecuada que permita relacionar de modo coherente la estructura matemática de la teoría con los fenómenos. En ese sentido, debemos remarcar el hecho de que, más de un siglo después de su nacimiento, no existe hoy un concepto capaz de dar cuenta en forma acabada de qué significa un vector en el espacio de Hilbert. En particular, cada vez que se consideran sistemas cuánticos compuestos, el resultado es que el espacio de estados se "extiende" al conjunto de matrices densidad. Si la interpretación de $|\psi\rangle$ es ya de por si un proble- 
UN NUEVO ESQUEMA CONGEPTUAL PARA LA INTERPRETACIÓN...

ma extremadamente complejo, las cosas se vuelven aún más complicadas para el caso de las matrices densidad. Puesto que carecemos de una definición adecuada de la noción de objeto en la mecánica cuántica y antes que frente a una estructura reduccionista parecemos encontrarnos ante una teoría holista (cf. Healey, 2002), el problema de los "sistemas cuánticos compuestos" aparece, referido a la estructura formal, como un verdadero oximoron. En lo que sigue, utilizaremos por comodidad el léxico corrientemente utilizado en la literatura sin dejar de señalar la carga interpretativa que conlleva esa discursividad.

Comúnmente se considera la relación entre los estados de un sistema copuesto y los estados de los subsistemas del siguiente modo. Enfoquémonos para mayor simplicidad en el caso de dos sistemas, $\mathrm{S}_{1}$ y S. $\mathrm{Si}\{|\mathrm{X}(1)\rangle\}$ y $\{|\mathrm{X}(2)\rangle\}$ son las bases ortonormales correspondientes de $\mathrm{H}_{1} \mathrm{y} \mathrm{H}_{2}$ respectivamente, entonces el conjunto $\{|\mathrm{x}(1)\rangle \otimes|\mathrm{x}(2)\rangle\}$ forma una base ortonormal para $\mathrm{H}_{1} \otimes \mathrm{H}_{2}$. Un estado puro del compuesto puede ser escrito como:

$$
\rho=|\psi\rangle\langle\psi|,
$$

donde $|\psi\rangle=\Sigma \alpha_{\mathrm{ij}}|\mathrm{x}(1)\rangle \otimes|\mathrm{x}(2)\rangle$. Si M representa un observable, su valor medio $\langle\mathrm{M}\rangle$ será dado por

$$
\operatorname{tr}(\rho M)=\langle M\rangle .
$$

Si los observables de la forma $\mathrm{O}_{1} \otimes_{1_{2}}$ y $1_{1} \otimes \mathrm{O}_{2}\left(\operatorname{con} \mathbf{1}_{1}\right.$ y $\mathbf{1}_{2}$ la identidad de los operadores sobre $\mathrm{H}_{1}$ y $\mathrm{H}_{2}$ respectivamente) son considerados, entonces los operadores de traza parcial $\rho_{1}$ y $\rho_{2}$ pueden ser definidos para sistemas $S_{1}$ y $S_{2}$. La relación entre $\rho, \rho_{1}$ y $\rho_{2}$ se encuentra dada por

$$
\rho_{1}=\operatorname{tr}_{2}(\rho), \quad \rho_{2}=\operatorname{tr}_{1}(\rho),
$$

donde tri significa la traza parcial sobre los $i$ grados de libertad. Puede ser demostrado que

$$
\begin{aligned}
& \operatorname{tr}_{1}\left(\rho_{1} O_{1} \otimes 1_{2}\right)=\left\langle O_{1}\right\rangle \\
& \operatorname{tr}_{2}\left(\rho_{2} 1_{1} \otimes O_{2}\right)=\left\langle O_{2}\right\rangle
\end{aligned}
$$

Operadores de la forma $\mathrm{O}_{1} \otimes \mathbf{1}_{2} \mathrm{y}_{1} \otimes \mathrm{O}_{2}$ representan magnitudes relacionadas a $\mathrm{S}_{1}$ y $\mathrm{S}_{2}$ respectivamente. Cuando $S$ es un estado producto $\left|\varphi_{1}\right\rangle \otimes\left|\varphi_{2}\right\rangle$, el valor medio del operador producto $\mathrm{O}_{1} \otimes \mathrm{O}_{2}$ vendrá dado por 


$$
\operatorname{tr}\left(\left|\varphi_{1}\right\rangle \otimes\left|\varphi_{2}\right\rangle\left\langle\varphi_{1}\right| \otimes\left\langle\varphi_{2}\right| O_{1} \otimes O_{2}\right)=\left\langle O_{1}\right\rangle\left\langle O_{2}\right\rangle,
$$

reproduciendo la independencia estadística.

Las mezclas son representadas por operadores positivos, hermíticos y de traza uno, también llamados "matrices densidad". El conjunto de todas las matrices densidad forma un conjunto convexo de estados que denominaremos $C$. Notar que los observables físicos se encuentran representados por elementos de $A$, el espacio vectorial$\mathrm{R}$ de los operadores hermíticos actuando sobre $\mathrm{H}$ :

$$
\begin{array}{ll}
\text { Definición 3.1 } & A:=\left\{A \in B(H) \mid A=A^{\dagger}\right\} \\
\text { Definición } 3.2 & \mathrm{G}:=\{\rho \in A \mid \operatorname{tr}(\rho)=1, \rho \geq 0\},
\end{array}
$$

donde $\mathrm{B}(\mathrm{H})$ aparece para el álgebra de operadores acotados en $\mathrm{H}$. El conjunto de estados puros satisface

$$
P:=\left\{\rho \in C \mid \rho^{2}=\rho\right\}
$$

Ese conjunto se encuentra en correspondencia con los rayos $\mathrm{H}$ por la asociación canónica

$$
\mathrm{F}: \mathrm{CP}(\mathrm{H}) \rightarrow \mathrm{C} \mid[|\psi\rangle] \rightarrow|\psi\rangle\langle\psi|,
$$

donde $\mathrm{CP}(\mathrm{H})$ es el espacio proyectivo de $\mathrm{H}$, y $[|\psi\rangle]$ es la clase definida por el vector $|\psi\rangle(|\varphi\rangle \sim|\psi\rangle \leftrightarrow \rightarrow|\varphi\rangle=\lambda|\psi\rangle, \lambda \neq 0)$. Ces el conjunto convexo dentro del hiperplano $\{\rho \in A \mid \operatorname{tr}(\rho)=1\}$ formado por la intersección de los hiperplanos con el cono positivo de matrices (ver Fig. 1).

Figura 1. Representación geométrica de la intersección entre el cono de matrices positivas y el hiperplano de traza igual a uno, que da lugar al convexo de estados cuánticos $C$. En el dibujo, se muestra también el conjunto de estados separables $S(H)$.

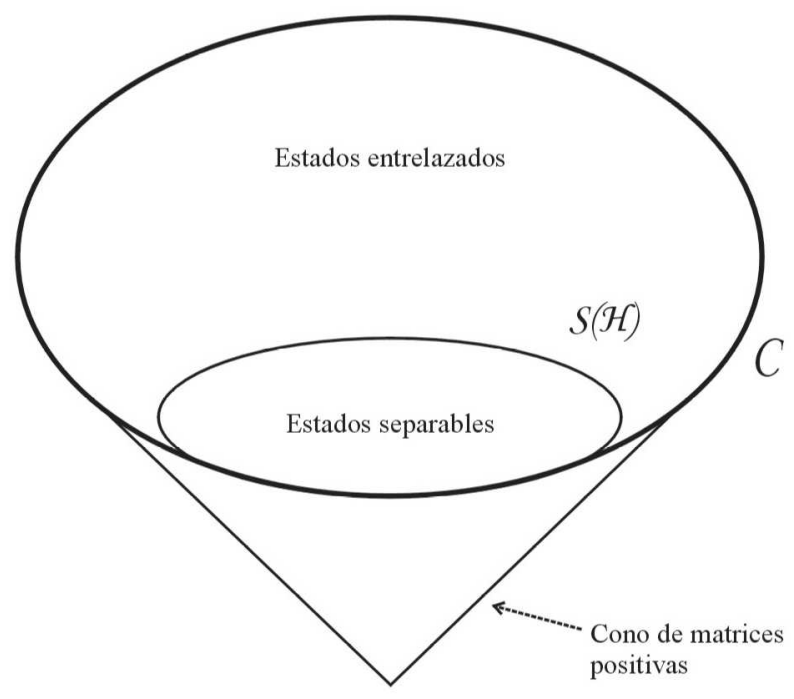


UN NUEVO ESQUEMA CONGEPTUAL PARA LA INTERPRETAGIÓN...

Los estados separables se encuentran definidos (cf. Werner, 1989) como aquellos estados de $C$ que pueden ser escritos como una combinación convexa de estados producto

$$
\rho_{\text {Sep }}=\Sigma \lambda_{\mathrm{ij}} \rho^{(1)} \otimes \rho^{(2)},
$$

donde $\rho^{(1)} \in C_{1}$ y $\rho^{(2)} \in C_{2}, \Sigma \lambda_{i j}=1$ y $\lambda_{\mathrm{ij}} \geq 0$. El conjunto $S(H)$ de estados separables se encuentra entonces definido por

\section{Definición $3.3 \quad S(H):=\{\rho \in G \mid \rho$ es separable $\}$}

Es un hecho remarcable el que existan estados que no se puedan factorizar como productos ni tampoco se puedan escribir como combinación convexa de estados producto. Si el estado no es separable, se dice, entonces, que se encuentra entrelazado (cf. Bengtsson \& Zyczkowski, 2006).

En mecánica estadística clásica, las mezclas no representan el grado de ignorancia respecto del estado actual, representado en el espacio de fases, en que se presupone se encuentra el sistema. En mecánica cuántica, por el contrario, debemos tener en cuenta, como sepala D’Espagnat (cf. 1976, cap. 6), la distinción entre mezclas propias y mezclas impropias. Una mezcla propia puede ser considerada como una matriz densidad a la que debemos sumarle una porción de información clásica que codifica probabilidades clásicas de ensambles de estados puros. Esa pieza "extra" de información clásica puede tener su origen en imperfecciones del procedimiento de preparación, o bien ser producida en forma deliberada. Las probabilidades encontradas en las mezclas propias pueden ser interpretadas en términos de ignorancia. Por el contrario, en el caso de las mezclas impropias no existe una tal información "extra" y no podemos considerar que las mezclas impropias nos brindan una información parcial del estado de cosas que puede ser suplementado por un conjunto de variables ocultas.

Una de las principales consecuencias de que existan estados no separables y de que sus matrices reducidas no puedan ser interpretadas en términos de ignorancia es que vale además el principio de superposición de estados.

$$
\begin{aligned}
& \text { Principio } 3.4 \text { (De SUPerposición) } \\
& \text { Si }\left|\psi_{1}\right\rangle \text { y }\left|\psi_{2}\right\rangle \text { son estados físicos, entonces } \alpha\left|\psi_{1}\right\rangle+\beta\left|\psi_{1}\right\rangle\left(|\alpha|^{2}+|\beta|^{2}=1\right) \\
& \text { también serán estados fisicos. }
\end{aligned}
$$

En el caso de las matrices densidad, podemos también construir combinaciones convexas de estados y referirnos a un principio de superposición análogo (cf. Holik, 2010), que llamaremos principio mezcla. 


\author{
Principio 3.5 (MEzGla) \\ Si $\rho$ y $\rho_{2}$ son estados físicos, entonces $\alpha \rho+\beta \rho^{\prime}(\alpha+\beta=1, \alpha, \beta \geq 0)$ \\ también serán estados físicos.
}

El principio mezcla aparece como una consecuencia directa de que el conjunto de estados cuánticos más general, es decir, el que incluye a todas las matrices densidad, es convexo. Mientras que para los estados puros siempre existen "proposiciones verdaderas" (cf. Piron, 1976), proposiciones para las que un experimento dará con certeza la respuesta “si” (o sea, una descomposición del vector en el que puede ser escrito como un término singular), la situación es radicalmente diferente para el caso de las mezclas impropias. Si aceptamos que las mezclas impropias son estados físicos tanto como lo son los estados puros, debemos enfrentarnos al hecho de que hay estados físicos para los que no existe ninguna "proposición verdadera" (dejando de lado la proposición trivial en la que la proposición representa el espacio de Hilbert). Como veremos en las próximas secciones, eso conlleva graves dificultades para mantener la interpretación ortodoxa de la lógica cuántica.

También debemos tomar en cuenta que vale, en general, para las matrices densidad que representan estados no-puros una ecuación análoga a 2.0.2,

$$
\rho=\Sigma p_{i} \rho_{\mathrm{i}}=\Sigma p_{i} \rho_{\mathrm{i}}^{\prime}=\Sigma p_{i}{ }^{\prime} \rho_{\mathrm{i}}{ }^{\prime}=\ldots
$$

La interpretación de la equivalencia entre cada uno de esos términos no resulta en modo alguno trivial, si queremos ir más allá de una interpretación instrumentalista que conciba a las diferentes descomposiciones de $\rho$ como un algoritmo que brinda las probabilidades para obtener resultados experimentales determinados.

\title{
3 RELAGIONES LÓGIGAS ENTRE MEZGLAS IMPROPIAS
}

Una forma de estudiar la estructura lógica de la mecánica cuántica es considerar la estructura que forman las proposiciones referidas a los experimentos del tipo "si-no", representados en mecánica cuántica por proyectores. Un proyector $\mathrm{P} \in \mathrm{B}(\mathrm{H})$ cumple

$$
\mathrm{P}^{2}=\mathrm{P}
$$

Denotaremos por " $P(H)$ " al conjunto de proyectores del espacio de Hilbert H. Es bien sabido que $P(H)$ se puede poner en correspondencia biyectiva con los sub-espacios de H. Usando la suma directa de sub-espacios como disyunción, la intersección de sub- 
espacios como conjunción y el complemento ortogonal como negación, $P(H)$ adquiere una estructura de retículo orto-modular (cf. Birkhoff \& von Neumann, 1936; Dalla Chiara, Giuntini \& Grichie, 2004; Piron, 1976; Rédei, 1998). Denotaremos al retículo de la mecánica cuántica por "LvN". Una de las principales características de los retículos ortomodulares es que, contrariamente a la mecánica clásica, pueden ser no distributivos, como en el caso de $P(H)$. Como es bien sabido, mientras que para un sistema clásico el retículo proposicional (LCM) que se obtiene resulta booleano (distributivo), para la mecánica cuántica, el álgebra del retículo de proposiciones resulta no distributivo. El enfoque lógico nos permite, considerando las proposiciones de experiencias físicas, dar cuenta de las diferencias de estructura lógica entre la mecánica clásica y la mecánica cuántica. Sin embargo, cuando se estudian sistemas cuánticos compuestos, la formulación de la lógica cuántica no parece poder dar cuenta de la relación entre sistemas y subsistemas (cf. Domenech, Holik, Massri, 2010; Holik, 2010; Holik, Massri, Ciancaglini, 2012).

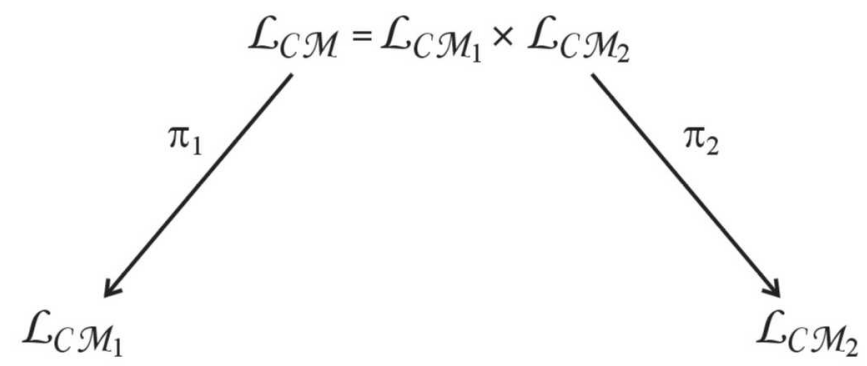

Figura 2. Para la mecánica clásica es posible usar las proyecciones conjuntistas $ð_{1}$ y $_{2}$ para conectar estados del sistema compuesto con sus subsistemas.

Para un sistema clásico compuesto, formado por dos subsistemas con retículos booleanos LCM y LCM $_{2}$ es posible usar las flechas ð $i$ que aparecen en la figura 2 de forma tal de conectar estados del sistema compuesto con estados de los subsistemas. Eso es posible, dado que en mecánica clásica se cumple la relación:

$$
\{s\}=\wedge\{p \in \operatorname{LGM} \mid p \text { es actual }\}
$$

la cual conecta el estado s con la conjunción de las propiedades (p) actuales del sistema, entendidas como aquellos experimentos que darán lugar a la respuesta "si" con certeza (o sea, con probabilidad igual a uno). Por el contrario, para el caso de la mecánica cuántica, resulta problemático establecer la conexión entre los espacios que su- 
puestamente representan subsistemas y aquellos que representan al sistema total. En este sentido no es posible dar cuenta de los símbolos “?” de la figura 3 que relacionan los retículos orto-modulares $\mathrm{LvN}, \mathrm{LvN}_{1}$ y $\mathrm{LvN}_{2}$ con mapas análogos a los del caso clásico.

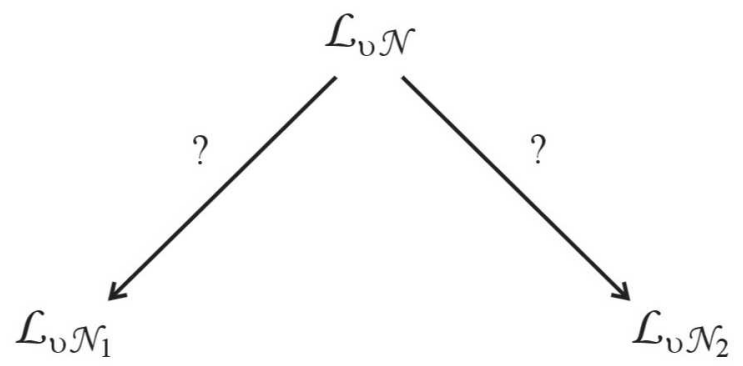

Figura 3. No se pueden aplicar trazas parciales para conectar estados de $\mathrm{LvN}$ con estados de $\mathrm{LvN}_{1} \mathrm{LLVN}_{2}$.

Esa imposibilidad de relacionar el sistema total y los subsistemas se debe principalmente a que los estados cuánticos se encuentran entrelazados. Las matrices densidad deben ser consideradas en tanto mezclas impropias y, por lo tanto, como sepalamos en la sección anterior, ya no existirán necesariamente experimentos que den la respuesta "si" con certeza. Eso hace que el enfoque lógico cuántico ortodoxo resulte particularmente inapropiado para la descripción de los llamados "sistemas compuestos".

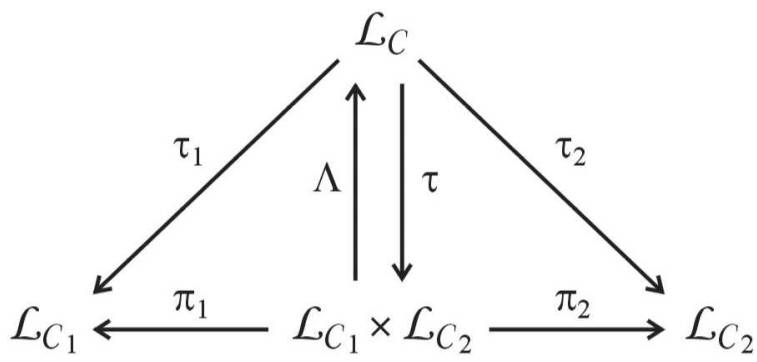

Figura 4. Los diferentes mapas entre $\mathrm{LC}_{1}, \mathrm{LC}_{2}, \mathrm{LC}_{1} \times \mathrm{LC}_{2} \mathrm{yLC}$.

Domenech, Holik y Massri (cf. 2010) han estudiado una reformulación del abordaje lógico cuántico que se basa en las mezclas impropias y en la estructura convexa de $C$ en lugar de $\mathrm{P}(\mathrm{H})$, que es la estructura formal en la cual se funda el enfoque ortodoxo. Tomando en cuenta los estados convexos, es posible dar cuenta de los mapas que relacionan el sistema total con los subsistemas. Mostramos los mapas en la figura 4, junto 
UN NUEVO ESQUEMA CONGEPTUAL PARA LA INTERPRETAGIÓN...

con los retículos basados en $C, L C_{1}, L C_{2}$ y $L C$. Esos diagramas permiten configurar una representación precisa de la estructura de relaciones entre las distintas descomposiciones del estado cuántico. A diferencia de los mapas análogos en mecánica clásica, en mecánica cuántica se tiene que $\Lambda \circ \tau \neq \tau \circ \Lambda$. La no conmutatividad entre $\Lambda$ y $\tau$ es una característica distintiva de dichas relaciones, y puede considerarse una expresión lógica-algebraica del entrelazamiento.

\section{Mezclas impropias E INTERPRETACión}

Evidentemente la estrategia interpretacional juega un papel fundamental a la hora de seguir los múltiples caminos que se despliegan y determinan la definición misma de qué significa el problema de interpretar la mecánica cuántica. En ese sentido, hemos sepalado que nuestra estrategia debe enmarcarse en aquél grupo de desarrollos e intentos que buscan ir, partiendo de la estructura formal de la teoría, hacia una interpretación conceptual adecuada que permita dar cuenta de modo coherente de la experiencia que encontramos en el laboratorio. Si bien la lógica cuántica podría ubicarse dentro de esta estrategia interpretativa, debemos también considerar atentamente el rol de la noción de actualidad como presupuesto metafísico. En el esquema de la física clásica, es posible considerar objetos materiales en el espacio-tiempo que respetan una evolución causal donde, instante a instante, cada sistema físico puede ser descripto a partir de sus propiedades actuales. El conjunto de los valores de las propiedades que caracterizan al sistema - las propiedades actuales que lo determinan - puede ponerse en relación con un punto en el espacio de las fases, que es el objeto matemático en el que se representan los estados físicos del sistema. La mecánica clásica nos dice, vía la ecuación de evolución, cómo cambia el sistema a partir de las condiciones iniciales. En otras palabras, la evolución del estado del sistema está representada por una curva en el espacio de las fases que determina cuáles son las propiedades actuales y cuáles las propiedades potenciales (cf. de Ronde, Freytes \& Domenech, en prensa). Sin embargo, puesto que la descripción física se encuentra completamente determinada por el conjunto de propiedades actuales, la posibilidad o potencialidad, en términos ontológicos, ocupa un lugar accesorio. En forma análoga, la lógica cuántica se desarrolla a partir de la determinación de los estados cuánticos por medio de las denominadas "propiedades actuales" que caracterizan al sistema. Esa idea se fundamenta en que, para estados puros, existe siempre una base en la que el vector puede ser escrito como un único término $(|\psi\rangle)$. El proyector asociado a ese vector $\mathrm{P}_{\psi}=|\psi\rangle\langle\psi|$ caracteriza, entonces, a la propiedad actual del sistema (o sea, aquella propiedad que el sistema posee con certeza) mientras que las demás propiedades son denominadas potenciales (o sea, 
aquellas propiedades que el sistema posee con probabilidad $p, 0<p<1$ ). Sin embargo, tomando en cuenta tan sólo el formalismo de la mecánica cuántica, no parece existir razón alguna para considerar, dentro de los estados puros, una base preferencial que caracterice al sistema en su modo de existencia (cf. de Ronde, 2011). Tampoco parece haber razón para tratar a los estados puros como elementos más primitivos o fundamentales que las matrices densidad, siendo los primeros un caso particular de los segundos. Si prestamos atención al formalismo y consideramos a las matrices densidad como la forma más general de caracterizar a los estados, los estados puros aparecen tan sólo como casos particulares de los estados cuánticos y ya no es posible-puesto que las mezclas cuánticas carecen, en principio, de toda propiedad actual - desarrollar la interpretación ortodoxa de la lógica cuántica.

Evidentemente, para avanzar en una interpretación que vaya más allá de los resultados experimentales, debemos comprender la estructura de elementos formales de la teoría que permiten acceder a la predicción de dichos eventos. Si es que estamos preparados para avanzar en una interpretación de las mezclas cuánticas dejando de lado los presupuestos clásicos, la lógica cuántica convexa nos demuestra que es posible considerar la estructura lógica de las relaciones entre matrices densidad en términos generales (o sea, sin la necesidad de que sean estados puros). Evadir la importancia de los estados mezcla deja también de lado los experimentos realizados en el presente en los laboratorios. Como es sabido, debido a la imposibilidad práctica de eliminar el ruido-ambiente, dicha experiencia cuántica se realiza tomando en cuenta, en la gran mayoría de los casos, estados mezcla y no estados puros (cf. Horodecki; Horodecki \& Horodecki, 2001). Los estados puros, aparecen como un límite ideal, en muchos casos, muy difícil de realizar en la práctica. De ese modo, tanto la estructura formal como la experiencia en los laboratorios nos arrastran a considerar las probabilidades que aparecen en las mezclas cuánticas más allá de la interpretación gnoseológica de la física clásica. Es posible pensar en un camino para desarrollar tal esquema en términos ontológicos (cf. de Ronde, 2011; 2012).

Otro de los elementos que permiten configurar la estructura formal de aquello que debe ser interpretado es la consideración de los diferentes niveles en que se representan las múltiples predicciones de los resultados. En el caso de las matrices densidad de la mecánica cuántica, las múltiples representaciones incompatibles, $\rho=\Sigma p_{i} \rho_{\mathrm{i}}$ $=\Sigma p_{i}{ }^{\prime} \rho_{\mathrm{i}}{ }^{\prime}=\Sigma p_{i}{ }^{\prime \prime} \rho_{\mathrm{i}}{ }^{\prime \prime}=\ldots$, determinadas por las distintas bases, nos brindan todas ellas conocimiento de aquello que pareciera debe ser considerado por la teoría como el "estado de cosas" descripto. Debemos remarcar en este sentido que debe distinguirse entonces claramente - debido a la incompatibilidad entre dichas bases - entre la representación de cada una de las bases y aquella que unifica las mismas en términos abstractos. Llamaremos a dicho estado de cosas, el cual engloba todas esas representa- 
ciones mutuamente incompatibles pero complementarias, $\rho^{\sim}$, y distinguiremos este símbolo de las matrices densidad escritas en las bases particulares $\rho, \rho^{\prime}, \rho^{\prime \prime}, \ldots$ Nos parece interesante investigar la posibilidad de interpretar dicho "estado de cosas" $\rho^{\sim}$ como la relación variacional entre la multiplicidad de resultados discretos (expuestos en cada una de las representaciones particulares):

$$
\rho^{\sim} \simeq \ldots \Sigma p_{i} \rho_{\mathrm{i}} \sim \Sigma p_{i}{ }^{\prime} \rho_{\mathrm{i}}{ }^{\prime} \sim \Sigma p_{i}{ }^{\prime \prime} \rho_{\mathrm{i}}{ }^{\prime \prime} \sim \ldots \quad \quad(5 \cdot 0.15)
$$

Cada una de las bases determina unívocamente un conjunto de predicciones bien definido, el "estado de cosas" $\rho$ " es el símbolo o la expresión que engloba las mutuas representaciones complementarias e incompatibles, determinadas por las matrices densidad escritas en las diferentes bases. Evidentemente $\rho^{\sim}$ excede las predicciones determinadas por una base singular, las unifica tomando en cuenta la incompatibilidad; $\rho$ contiene toda la información físicamente disponible para todos los experimentos concebibles, los cuales incluyen contextos mutuamente incompatibles (por ejemplo, tomografías cuánticas). La pregunta que debe ser respondida es qué tipo de concepto puede dar cuenta de tal estructura fenoménica y formal. Esperamos que al continuar con el trayecto aquí expuesto nos encaminemos hacia un nuevo esquema conceptual que nos permita interpretar, en términos de la realidad física, las matrices densidad en la mecánica cuántica.@

Agradecimientos. Este trabajo fue sostenido por las siguientes becas: PIP No 6461/o5 (CON- ICET) y el Proyecto FWO G.04.0508N de la Vrije Universiteit Brussel. Queremos agradecer a Graciela Domenech por comentarios a una versión anterior.

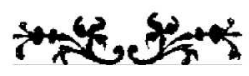


Federico Holix Investigador post-doctoral del Instituto de Física de La Plata, Universidad de Buenos Aires, Argentina. holik@fisica.unlp.edu.ar

\title{
Christian DE RONDE
}

Instituto de Filosofía, Universidad de Buenos Aires, Investigador del Consejo Nacional de Investigaciones

Científicas y Tecnológicas, Argentina. Investigador afiliado del Center Leo Apostel, Foundations of the Exact Sciences, Vrije Universiteit Brussel, Bélgica. cderonde@vub.ac.be

Wim Christiaens Investigador del Center Leo Apostel, Vrije Universiteit Brussel, Bélgica. wim.a.christiaens@gmail.com

\section{A new conceptual scheme for the interpretation of the improper mixtures in quantum mechanics}

\begin{abstract}
In this article, we analyze the meaning of density matrices within the formalism of quantum mechanics. We discuss the problem of compound systems in the context of quantum logic as well as the interpretation of improper mixtures. Taking into account the development of convex quantum logic, we present an analysis of the formal structure of the theory which we will argue, must be taken into account when developing a new conceptual scheme for the interpretation of quantum mixtures.
\end{abstract}

KeYwords • Quantum logic. Improper mixtures. Convex sets. 
UN NUEVO ESQUEMA GONGEPTUAL PARA LA INTERPRETAGIÓN...

\section{REFERENGIAS BIBLIOGRÁFIGAS}

Alber, G. et al. (Ed.). Quantum information. Berlin: Springer, 2001. (Springer Tracts in Modern Physics, 173.)

Bengtsson, I. \& Zyczkowski, K. Geometry of quantum states: an introduction to quantum entanglement. Cambridge: Cambridge University Press, 2006.

Birkhoff G. \& von Neumann, J. The logic of quantum mechanics. Annals of Mathematics, 37, p. 823-43, 1936.

Bттвоц, M. Mechanique quantique, une introduction philosophique. Paris: Champs Flamarion, 1997.

Вонм, D. Asuggested interpretations of the quantum theory in terms of "hidden variables". Part I. Physical Review, 85, p. 166-79, 1952a.

. A suggested interpretations of the quantum theory in terms of "hidden variables". Part II. Physical Review, 85, p. 180-93, 1952b.

Boкulich, A. Open or closed? Dirac, Heisenberg, and the relation between classical and quantum mechanics. Studies in History and Philosophy of Modern Physics, 35, p. 377-96, 2004.

Dalla Chiara, M. L.; Giuntinni, R. \& Greechie, R. Reasoning in quantum theory. Dordrecht: Kluwer Academic Publishers, 2004.

DE Ronde, C. El enfoque de descripciones complementarias: en búsqueda de un desarrollo expresivo de la realidad física. Perspectivas Metodológicas, 9, p. 126-38, 2009.

. For and against metaphysics in the modal interpretation of quantum mechanics. Philosophica, 83, p. $85^{-117}, 2010$.

. The contextual and modal character of quantum mechanics. Utrecht, 2011. Tesis (Doctorado en Física). Utrecht University.

. La noción de potencialidad en la interpretación modal de la mecánica cuántica. Scientiae Studia, 10, p. $137-64,2012$.

De Ronde, C. \& Bontems, V. La notion d'entité en tant qu'obstaclé épistémologique: Bachelard, la mécanique quantique et la logique. Bulletin des Amis de Gaston Bachelard, 13, p. 12-38, 2011.

de Ronde, C. \& Fleisner, A. Sobre teorías cerradas y paradigmas: un dialogo entre Werner Heisenberg y Thomas Kuhn. Actas del ıer Congreso de la Sociedad Filosófica Uruguaya, 2012.

de Ronde, C.; Freytes, H \& Domenech, G., Interpreting the modal Kochen-Specker theorem: possibility and many worlds in quantum mechanics. En prensa.

D’Espagnat, B. Conceptual foundations of quantum mechanics, Massachusetts: Benjaming Reading, 1976. Reply to K. A. Kirkpatrick. ArXiv: quant-pho111081, 2001.

Dickson, M. \& Dieks, D. Modal interpretations of quantum mechanics. In: ZaLta, E. N. (Ed.). The Stanford Encyclopedia of Philosophy, 2002. Disponible en: <http://plato.stanford.edu/archives/ win2002/ entries/qm-modal/>. Acceso en: 20 oct. 2012.

Dirac, P. A. M. The principles of quantum mechanics. 4 ed. London: Oxford University Press, 1974.

Domenech, G., Holik, F. \& Massri, C. A quantum logical and geometrical approach to the study of improper mixtures. Journal Mathematical Physics, 51, p. 521081-7, 2010.

Fine, A. The shaky game. Chicago: University of Chicago Press, 1986.

Fuchs, C. \& Peres, A. Quantum theory needs no "interpretation". Physics Today, 53, p. 70-2, 2000.

Ghirardi, G. G., Rimini, A. \& Weber, T. Unified dynamics for microscopic and macroscopic systems. Physical Review D, 34, p. 470-91, 1986.

Healey, R. Holism and nonseparability in physics. In: Zatta, E. N. (Ed.). The Stanford Encyclopedia of Philosophy, 2002. Disponible en: <http://plato.stanford.edu/entries/physicsp-holism/>. Acceso en: 20 oct. 2012. 
Holik, F. Compound quantum systems: an algebraic approach. Buenos Aires, 2010. Tesis (Doctorado en Física). Universidad de Buenos Aires.

Holik, F.; Massri, G. \& Ciancaglini, N. Convex quantum logic. International Journal of Theoretical Physics, 51, p. $1600-20,2012$.

Horodecki, M.; Horodecki, P. \& Horodecki, R. Mixed-state entanglement and quantum communication. In: Alber, G. et al. (Ed.). Quantum information. Berlin: Springer, 2001. (Springer Tracts in Modern Physics, v. 173.). p. $15^{1-6} 5$.

Howard, D. Was Einstein really a realist? Perspectives on Science, 1, p. 204-51, $199^{3}$.

. Einstein, Kant, and the origins of logical empiricism. In: Salmon, W. \& Wolters, G. (Ed.). Logic, language, and the structure of scientific theories. Pittsburgh: University of Pittsburgh Press, 1994 . (Proceedings of the Carnap-Reichenbach centennial, University of Konstanz, 21-24, May 1991.). p. $45^{-105}$.

JaUch. J. M. Foundations of quantum mechanics. Cambridge: Addison-Wesley, 1968.

Kochen, S. Anew interpretation of quantum mechanics. In: Lathi, P. \& Mittelslaedt, P. (Ed.). Symposium on the foundations of modern Physics 1985. Johensuu: World Scientific, 1985· p. 151-69.

Kochen, S. \& Spegker, E. On the problem of hidden variables in quantum mechanics. Journal of Mathematics and Mechanics, 17, p. 59-87, 1967.

Lathi, P. \& Mittelslaedt, P. (Ed.). Symposium on the foundations of modern Physics 1985. Johensuu: World Scientific, 1985 .

Mittelstaedt, P. The interpretation of quantum mechanics and the measurement process. Cambridge: Cambridge University Press, 1998.

Piron, C. Foundations of quantum physics, Cambridge: Addison-Wesley, 1976.

RÉDEI, M. Quantum logic in algebraic approach. Dordrecht: Kluwer Academic Publishers, 1998.

Salmon, W. \& Wolters, G. (Ed.). Logic, language, and the structure of scientific theories. Pittsburgh: University of Pittsburgh Press, 1994. (Proceedings of the Carnap-Reichenbach centennial, University of Konstanz, 21-24, May 1991.)

Van Fraassen, B. C. Quantum mechanics: an empiricist view, Oxford: Clarendon, 1991.

Werner, R. Quantum states with Einstein-Podolsky-Rosen correlations admitting a hidden-variable model. Physical Review A, 4, p. p. 4277-81, 1989.

Zatta, E. N. (Ed.). The Stanford Encyclopedia of Philosophy. Standford: Standford University Press, 2008. $10 \mathrm{~V}$.
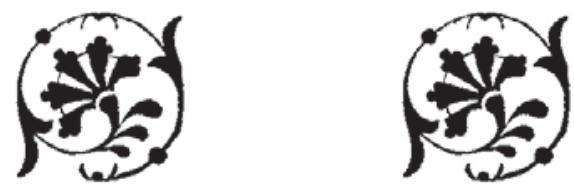